\title{
Frequency and intensity of gastrointestinal symptoms in exercisers individuals at rest and during physical exercise: an internet-based survey
}

\author{
Claudio Andre Barbosa de Lira ${ }^{1}$, Ricardo Borges Viana ${ }^{1}$, Kaylla Priscilla Mesquista $^{2}$, Douglas de Assis Teles Santos ${ }^{1,3}$, \\ Mário Hebling Campos ${ }^{1}$, Marília Santos Andrade ${ }^{4}$, Rodrigo Luiz Vancini ${ }^{5}$ \\ ${ }^{1}$ Human and Exercise Physiology Sector, Human Movement Assessment Laboratory, Faculty of Physical Education and Dance, Federal \\ University of Goiás, Goiânia; ${ }^{2}$ Special Academic Unit of Health Sciences, Federal University of Jatai, Jatai, ${ }^{3}$ Collegiate of Physical Education, \\ Bahia State University, Teixeira de Freitas; ${ }^{4}$ Department of Physiology, Federal University of São Paulo, São Paulo; ${ }^{5}$ Center of Physical Education \\ and Sports, Federal University of Espirito Santo, Vitória, Brazil
}

Background/Aims: Despite the evidence of a modest to high prevalence of gastrointestinal (GI) symptoms in recreational runners and endurance athletes, the frequency and intensity of GI symptoms in exercisers, but nonathletes, individuals from different modalities have been less investigated. Therefore, the present study aimed to assess the prevalence of GI symptoms in individuals that practice moderate or vigorous physical exercise, at rest and during physical exercise training session. Methods: The sample consisted of 142 exercisers individuals (64 women and 78 men with mean age of $32.9 \pm 10.7$ years). Out of the 142 participants, 71 reported to perform moderate physical exercise and 71 reported to perform vigorous physical exercise. Participants were assessed by an internet-based questionnaire designed to assess the frequency and intensity (at rest and during physical exercise training session) of 18 GI symptoms. Results: The GI symptoms most frequently reported by the respondents (during rest and physical exercise training session, respectively) were flatulence (90.8\% and 69.7\%), abdominal noise (77.5\% and $41.5 \%)$, and eructation $(73.9 \%$ and $52.1 \%)$. Overall, the frequency and intensity of symptoms were higher $(P<0.050)$ during rest than physical exercise training session for who perform moderate and vigorous physical exercise. Conclusions: It can be concluded that GI symptoms in exercisers, but nonathletes, individuals are more prevalent during rest than during physical exercise training session, suggesting that moderate and vigorous physical exercise may act as a regulator of the GI tract. (Intest Res 2019;17:537-545)

Key Words: Gastrointestinal symptoms; Gastrointestinal tract; Exercise

\section{INTRODUCTION}

Physical exercise is widely recognized for its beneficial effects on cardiovascular, respiratory, and muscular systems. ${ }^{1,2}$ How-

Received December 10, 2018. Revised February 15, 2019

Accepted April 1, 2019

Correspondence to Claudio Andre Barbosa de Lira, Human and Exercise

Physiology Sector, Human Movement Assessment Laboratory, Faculty

of Physical Education and Dance, Federal University of Goiás, Avenida

Esperança s/n, Campus Samambaia, Goiânia 74690-900, Brazil.

Tel: +55-62-3521-1141, Fax:+55-62-3521-1141,E-mail: andre.claudio@

gmail.com ever, physical exercise also triggered effects on physiological systems which were not directly related to the exercise such as the GI system. ${ }^{3}$ Indeed, the impact of physical exercise on the GI system, both in experimental models and in humans, is an area of great interest. ${ }^{4-7}$ For example, moderate physical exercise may act as a modulator of GI function, because it may prevent diseases such as colon cancer, cholelithiasis, diverticulitis, IBD, and constipation..$^{5,8}$ On the other hand, high intensity and long duration physical exercise triggers GI symptoms, ${ }^{3,5,8,9}$ which may occur at rest or during physical exercise session., ${ }^{5,8,10-16}$ When these symptoms occur during physical exercise, they 
may impair an individual's ability to perform physical effort and result in interruption in severe cases. ${ }^{10,12,17}$ GI symptoms can be divided into upper (upper abdominal bloating, epigastric pain, vomiting, nausea, belching, heartburn, and loss of appetite) and lower (flatulence, diarrhea, abdominal cramps, bleeding, accelerated bowel movements, and urge to defecate). ${ }^{3}$

The prevalence of GI symptoms in elite endurance athletes, such as marathon runners, triathletes, and cyclists, has been well described in the literature and can attain rates of up to $90 \%$ depending on the methodology used and events studied. ${ }^{10}$ More specifically, the prevalence of diarrhea and accelerated bowel movements among marathoners is $38 \%$ and $53 \%$, respectively. ${ }^{17}$ The triggering of GI symptoms may be attributed to physiological, mechanical, or nutritional factors. ${ }^{3,18,19}$ During intense exercise, and especially in a hypohydrated state, mesenteric blood flow is decreased. ${ }^{10}$ This is believed to be one of the main contributors to the development of GI symptoms, since reduced splanchnic perfusion may result in compromised gut permeability in athletes. ${ }^{10}$ By different mechanisms, athletes that perform anaerobic sports such as weightlifting, which cause high levels of intra-abdominal pressure, also present GI symptoms, including heartburn and gastroesophageal reflux. ${ }^{20}$ It is noteworthy that GI symptoms may also affect the individual at rest state. ${ }^{16}$

Despite this knowledge available in the literature and evidence of a modest prevalence of GI symptoms in recreational runners, ${ }^{21}$ the frequency and intensity of GI symptoms in exercisers individuals (nonathletes) have been less investigated. Therefore, further studies to elucidate the prevalence of GI symptoms in exercisers people at rest and during physical exercise training session are needed. Thus, the aims of the present study were to investigate the frequency and intensity of GI symptoms, during rest and physical exercise training session, in individuals that practice different types of moderate or vigorous physical exercise for recreational and health purposes. It was hypothesized that exercisers, but nonathletes, present GI symptoms at rest and during exercise, akin to endurance athletes, however these symptoms are of moderate intensity given the lower intensity and duration of exercise performed by this population. In addition, it was hypothesized that intensity and frequency of these symptoms are greater during resting state as compared to physical exercise since is known that physical exercise acts as regulator of GI function. Finally, it was hypothesized that intensity and frequency of these symptoms are greater in individuals that performing vigorous physical exercise as compared with individuals that performing moderate physical exercise. A better understanding of the frequency and intensity of GI symptoms in this population may help guide exercise prescription and be useful in treatment and prevention programs for GI diseases and symptoms.

\section{METHODS}

\section{Participants}

This was a cross-sectional study of 142 trained individuals (78 men and 64 women). Participants from different states of Brazil were recruited over the internet. All participants were engaged in regular physical exercise programs of, at least, moderate intensity for at least 2 months. To the end of current study, we considered regular physical exercise that physical exercise performed for at least 2 months, since previous studies investigating training effects had found positive physiological adaptations with 2 months of training. ${ }^{22-24}$ Respondents under the age of 18, athletes, who performed physical exercise of very, very light; very light, and light intensity and who had GI diseases or other disease that influence GI function, who used medical drugs that influence GI function and/or were submitted to gastric or intestinal surgery were excluded. For the purposes of current study, we consider as athlete who participates in an organized team or individual sport that requires regular competition against others as a central component, places a high premium on excellence and achievement, and requires systematic training. ${ }^{25}$ The participants were divided in 2 groups: physical exercise of moderate intensity group (comprised by participants that reported to perform physical exercise of moderate intensity: $\mathrm{n}=71,37$ women) and physical exercise of vigorous intensity group (comprised by participants that reported to perform physical exercise of vigorous intensity: $n=71,27$ women). The characteristics of the participants are shown in Table 1. The Mann-Whitney $U$-test revealed significant differences between group for height, experience in physical exercise, weekly frequency of physical exercise, hours per day of physical exercise and intensity of physical exercise.

Participants were informed about the aims and procedures of the study by a text over the internet (available at https:// docs.google.com/spreadsheet/viewform?formkey = dExTTkp yaWJLWXIXdUZSMGIQcW5QMnc6MQ\#gid=0). Voluntary and full completion of the questionnaire was taken to indicate that the participants agreed with the objectives of the study and felt clearly informed about the experimental procedures. All experimental procedures were approved by the Federal 
Table 1. Characteristics of Participants $(n=142)$

\begin{tabular}{lccc}
\hline \multirow{2}{*}{ Variable } & \multicolumn{2}{c}{ Group } & \multirow{2}{*}{$\boldsymbol{P}$-value } \\
\cline { 2 - 3 } & Moderate $(\mathrm{n}=71)$ & Vigorous $(\mathrm{n}=71)$ & 0.442 \\
\hline Age $(\mathrm{yr})$ & $34.04 \pm 11.25$ & $31.66 \pm 10.07$ & 0.021 \\
Height $(\mathrm{m})$ & $1.69 \pm 0.09$ & $1.72 \pm 0.07$ & 0.185 \\
Body mass $(\mathrm{kg})$ & $70.39 \pm 15.23$ & $72.78 \pm 13.21$ & 0.977 \\
BMl $\left(\mathrm{kg} / \mathrm{m}^{2}\right)$ & $24.50 \pm 3.78$ & $24.43 \pm 3.60$ & 0.013 \\
Experience in physical exercise $(\mathrm{yr})$ & $7.22 \pm 8.47$ & $11.50 \pm 10.79$ & $<0.001$ \\
Weekly frequency of physical exercise & $3.46 \pm 1.30$ & $4.52 \pm 1.37$ & $<0.001$ \\
Physical exercise (hr/day) & $1.25 \pm 0.50$ & $1.67 \pm 0.87$ & $<0.001$ \\
Intensity of physical exercise & $4.00 \pm 0.00$ & $5.30 \pm 0.52$ & \\
\hline
\end{tabular}

Values are presented as mean \pm SD.

University of Goiás Human Research Ethics Committee (approval number: 066/2013) and conformed to the principles outlined in the Helsinki Declaration.

\section{Questionnaire}

A questionnaire was devised (available at https://docs.google. $\mathrm{com} /$ spreadsheet/viewform?formkey = dExTTkpyaWJLWXIX dUZSMGIQcW5QMnc6MQ\# gid =0), based on a thorough review of the specialized literature, to investigate the frequency and intensity of GI symptoms at rest and during physical exercise training session. The questionnaire comprised 3 parts: first, personal data and training information (age, body mass, height, gender, exercise modalities, intensity of training sessions, weekly training frequency [number of sessions per week], and length of each training session [in hours]); second, weekly frequency and intensity of GI symptoms in the resting state; and third, weekly frequency and intensity of GI symptoms during physical exercise. To answer on the frequency and intensity of GI symptoms during rest, the volunteers were instructed not to take into account the period of up to 3 hours after the end of the exercise. The following GI symptoms (in rest state and during physical exercise training session) were assessed: epigastric pain, regurgitation, nausea, vomiting, early satiety, dysphagia, bloating, eructation, abdominal noise, abdominal cramps (lower abdominal pain), blood in stools, diarrhea, constipation, steatorrhea, mucus stools, loss of appetite, and heartburn. In order to aid understanding, each symptom contained in the list was accompanied by the corresponding terms used by the lay population.

Likert type scales ${ }^{26}$ in the $1-7$ or 1-5 range were adopted to evaluate "intensity of the physical exercise training sessions" ( 1 , very, very light; 2 , very light; 3 , light; 4 , moderate; 5 , intense;
6, very intense; and 7, maximal), "weekly frequency index of GI symptoms" (1, indicated no GI symptoms; 2, rarely; 3, sometimes; 4, frequently; and 5, always), and "intensity index of GI symptoms" (1, indicated no GI symptoms; 2, very light; 3 , light; 4 , moderate; 5 , intense; 6 , very intense; and 7 , maximal). Likert scale to assess intensity of the physical exercise training session was created and adapted from American College of Sports Medicine guidelines. ${ }^{1}$ Again, participants that reported to perform very, very light; very light, and light physical exercise were excluded from sample. Participants that reported to perform intense, very intense, and maximal physical exercise were grouped in physical exercise of vigorous intensity group.

\section{Statistical Analysis}

Data were analyzed using the software application SPSS version 21.0 (IBM Corp., Armonk, NY, USA). Descriptive statistics were used to analyze the data (mean and SD, and absolute and relative frequencies). The absolute frequency of each GI symptom was calculated by adding up the number of individuals that reported the symptoms, i.e., those who marked 2, 3, 4, or 5 . Relative frequency of each GI symptom was calculated by dividing the absolute frequency by total number of individuals in the sample, expressed as a percentage. The McNemar test was used to evaluate the GI symptoms during rest and during physical exercise training session.

The Mann-Whitney $U$-test was used to compare variables between the groups (moderate and vigorous group). Additionally, frequency was evaluated by a "weekly frequency index of GI symptoms" which consisted of assessing the mean and standard deviation of the responses given in the questionnaire. The same procedure was adopted for intensity "intensity index of GI symptoms." These data were compared between 
groups using two-way ANOVA (group [moderate $\times$ vigorous] $\times$ state [rest $\times$ physical exercise]). The level of significance was $P<0.050$.

\section{RESULTS}

Of the 142 individuals investigated, 68 practiced resistance training, 64 running/walking, 18 swimming, 11 soccer, 14 cycling, 12 martial arts/fighting, 2 functional training, 5 volleyball, 6 triathlon, 5 Pilates, 4 tennis, 2 surfing, 2 yoga, 2 stretching, 2 dance, and 1 basketball. In addition, 82 practiced only 1 modality, 34 practiced 2 modalities, 18 practiced 3 modalities, 6 practiced 4 modalities, and 2 practiced 5 modalities. The average intensity of exercise reported during training session, considering all modalities, was $4.7 \pm 0.8$, which corresponded to an estimated moderate exercise intensity according to scale proposed by current study. Specifically, 71 (50\%) report that performed physical exercise of "moderate" intensity and 71 (50\%) report that performed "vigorous" physical exercise (Table 1).

Overall, the most prevalent symptoms were flatulence, abdominal noise, and eructation (Table 2). In addition, except for regurgitation, nausea, dysphagia, blood in stools, steatorrhea and loss of appetite, the McNemar test revealed a high proportion of GI symptoms in resting state compared with physical exercise training session.

To assess the frequency and intensity indexes of GI symptoms, we divided the sample into 2 groups (moderate physical exercise and vigorous physical exercise groups) according described in methods section. For frequency index of GI symptoms, the two-way ANOVA did not reveal state (rest $\times$ physical exercise) and group (moderate $\times$ vigorous) interaction for any GI symptoms $(P>0.050)$. However, two-way ANOVA revealed effect of state $(P>0.050$, lower weekly frequency index of GI symptoms during exercise for both groups) for following symptoms: epigastric pain, vomiting, early satiety, bloating, eructation, abdominal noise, abdominal cramps, flatulence, diarrhea, constipation and mucus in stools. Descriptive data about frequency index of GI symptoms of the participants are presented in Table 3. When data were analyzed together, weekly frequency index of GI symptoms were $1.61 \pm 0.38$ and $1.35 \pm 0.36$ at rest and at physical exercise training session, respectively, for moderate group and $1.70 \pm 0.30$ and $1.44 \pm 0.33$ at rest and at physical exercise training session, respectively, for vigorous group.

Regards to intensity index of GI symptoms, the two-way
Table 2. Absolute (and Relative) Frequency of GI Symptoms during Rest and Physical Exercise $(n=142)$

\begin{tabular}{lccr}
\hline Symptom & Rest & $\begin{array}{c}\text { Physical } \\
\text { exercise }\end{array}$ & $P$-value \\
\hline Epigastric pain & $46(32.4)$ & $28(19.7)$ & 0.001 \\
Regurgitation & $47(33.1)$ & $40(28.2)$ & 0.210 \\
Nausea & $44(31.0)$ & $36(25.4)$ & 0.115 \\
Vomiting & $10(7.0)$ & $1(0.7)$ & 0.004 \\
Early satiety & $77(54.2)$ & $46(32.4)$ & $<0.001$ \\
Dysphagia & $11(7.7)$ & $10(7.0)$ & 1.000 \\
Bloating & $91(64.1)$ & $42(29.6)$ & $<0.001$ \\
Eructation & $105(73.9)$ & $74(52.1)$ & $<0.001$ \\
Abdominal noise & $110(77.5)$ & $59(41.5)$ & $<0.001$ \\
Abdominal cramps & $58(40.8)$ & $31(21.8)$ & $<0.001$ \\
Flatulence & $129(90.8)$ & $99(69.7)$ & $<0.001$ \\
Blood in stools & $9(6.3)$ & $5(3.5)$ & 0.125 \\
Diarrhea & $52(36.6)$ & $37(26.1)$ & 0.003 \\
Constipation & $60(42.3)$ & $41(28.9)$ & $<0.001$ \\
Steatorrhea & $7(4.9)$ & $3(2.1)$ & 0.125 \\
Mucus in stools & $21(14.8)$ & $12(8.5)$ & 0.004 \\
Loss of appetite & $26(18.3)$ & $20(14.1)$ & 0.286 \\
Heartburn & $72(50.7)$ & $60(42.3)$ & 0.023 \\
\hline Valuesare pren & &
\end{tabular}

Values are presented as number (\%).

ANOVA did not reveal state (rest $\times$ physical exercise) and group (moderate $\times$ vigorous) interaction for any GI symptoms $(P>$ $0.050)$. However, two-way ANOVA revealed effect of state $(P>$ 0.050 , lower intensity index of GI symptoms during exercise for both groups) for following symptoms: epigastric pain, early satiety, bloating, eructation, abdominal noise, abdominal cramps, flatulence, diarrhea, constipation and mucus in stools. Descriptive data about intensity index of GI symptoms of the participants are presented in Table 4 . When data were analyzed together, intensity index of GI symptoms were $1.78 \pm 0.41$ and $1.46 \pm 0.40$ at rest and at physical exercise training session, respectively, for moderate group and $1.86 \pm 0.39$ and $1.54 \pm 0.37$ at rest and at physical exercise training session, respectively, for vigorous group.

\section{DISCUSSION}

Studies have shown that endurance physical exercise, such as that performed by marathoners, triathletes and cyclists can cause GI symptoms which may impair physical performance. ${ }^{10}$ However, there is little information on the frequency and in- 
Table 3. Comparison of Weekly Frequency Index of GI Symptoms during Rest and Physical Exercise

\begin{tabular}{|c|c|c|c|c|c|c|c|}
\hline \multirow{3}{*}{ Symptom } & \multicolumn{4}{|c|}{ Group } & \multirow{3}{*}{$\begin{array}{c}P \text {-value } \\
\text { (intensity) } \\
\text { (moderatex } \\
\text { vigorous) }\end{array}$} & \multirow{3}{*}{$\begin{array}{c}P \text {-value } \\
\text { (state) } \\
\text { (rest } \times \text { physical } \\
\text { exercise) }\end{array}$} & \multirow{3}{*}{$\begin{array}{c}P \text {-value } \\
\text { (interaction) } \\
\text { (intensity } \times \\
\text { state) }\end{array}$} \\
\hline & \multicolumn{2}{|c|}{ Moderate $(n=71)$} & \multicolumn{2}{|c|}{ Vigorous $(n=71)$} & & & \\
\hline & Rest & $\begin{array}{l}\text { Physical } \\
\text { exercise }\end{array}$ & Rest & $\begin{array}{l}\text { Physical } \\
\text { exercise }\end{array}$ & & & \\
\hline Epigastric pain & $1.54 \pm 0.97$ & $1.35 \pm 0.74$ & $1.55 \pm 0.84$ & $1.28 \pm 0.59$ & 0.766 & 0.018 & 0.655 \\
\hline Regurgitation & $1.55 \pm 0.88$ & $1.39 \pm 0.73$ & $1.56 \pm 1.04$ & $1.41 \pm 0.92$ & 0.895 & 0.147 & 1.000 \\
\hline Nausea & $1.38 \pm 0.66$ & $1.35 \pm 0.70$ & $1.45 \pm 0.82$ & $1.37 \pm 0.70$ & 0.241 & 0.429 & 0.107 \\
\hline Vomiting & $1.06 \pm 0.29$ & $1.00 \pm 0.00$ & $1.14 \pm 0.42$ & $1.03 \pm 0.24$ & 0.094 & 0.012 & 0.401 \\
\hline Early satiety & $2.11 \pm 1.24$ & $1.55 \pm 0.97$ & $2.23 \pm 1.30$ & $1.51 \pm 0.81$ & 0.787 & $<0.001$ & 0.552 \\
\hline Dysphagia & $1.08 \pm 0.37$ & $1.06 \pm 0.29$ & $1.18 \pm 0.66$ & $1.17 \pm 0.61$ & 0.080 & 0.726 & 0.907 \\
\hline Bloating & $1.93 \pm 0.90$ & $1.39 \pm 0.69$ & $2.04 \pm 0.95$ & $1.52 \pm 0.89$ & 0.243 & $<0.001$ & 0.945 \\
\hline Eructation & $2.32 \pm 1.18$ & $1.73 \pm 0.98$ & $2.42 \pm 1.30$ & $1.96 \pm 1.18$ & 0.242 & $<0.001$ & 0.647 \\
\hline Abdominal noise & $2.39 \pm 1.05$ & $1.65 \pm 0.90$ & $2.41 \pm 1.13$ & $1.72 \pm 1.02$ & 0.729 & $<0.001$ & 0.817 \\
\hline Abdominal cramps & $1.61 \pm 0.85$ & $1.28 \pm 0.64$ & $1.61 \pm 0.82$ & $1.34 \pm 0.65$ & 0.751 & 0.001 & 0.751 \\
\hline Flatulence & $2.70 \pm 1.18$ & $2.10 \pm 1.06$ & $3.20 \pm 1.06$ & $2.38 \pm 1.15$ & 0.004 & $<0.001$ & 0.425 \\
\hline Blood in stools & $1.06 \pm 0.29$ & $1.03 \pm 0.24$ & $1.11 \pm 0.52$ & $1.08 \pm 0.50$ & 0.244 & 0.560 & 1.000 \\
\hline Diarrhea & $1.45 \pm 0.75$ & $1.27 \pm 0.53$ & $1.56 \pm 0.84$ & $1.35 \pm 0.70$ & 0.246 & 0.021 & 0.868 \\
\hline Constipation & $1.70 \pm 1.07$ & $1.44 \pm 0.91$ & $1.68 \pm 0.94$ & $1.38 \pm 0.76$ & 0.701 & 0.011 & 0.898 \\
\hline Steatorrhea & $1.00 \pm 0.00$ & $1.00 \pm 0.00$ & $1.06 \pm 0.23$ & $1.03 \pm 0.17$ & 0.013 & 0.407 & 0.407 \\
\hline Mucus in stools & $1.14 \pm 0.35$ & $1.06 \pm 0.29$ & $1.15 \pm 0.44$ & $1.07 \pm 0.26$ & 0.727 & 0.037 & 1.000 \\
\hline Loss of appetite & $1.15 \pm 0.36$ & $1.15 \pm 0.47$ & $1.28 \pm 0.72$ & $1.18 \pm 0.52$ & 0.222 & 0.437 & 0.437 \\
\hline Heartburn & $1.82 \pm 1.06$ & $1.80 \pm 1.05$ & $1.93 \pm 1.10$ & $1.65 \pm 0.88$ & 0.862 & 0.226 & 0.273 \\
\hline
\end{tabular}

Values are presented as mean \pm SD.

tensity of GI symptoms in exercisers nonathletes individuals. Thus, the aim of this study was to evaluate the frequency and intensity of GI symptoms in a sample of exercisers nonathletes individuals at resting state and during physical exercise training session. The main result was that individuals present GI symptoms at rest and during physical exercise training session, akin to endurance athletes, however these symptoms are of very light intensity. In addition, the frequency and intensity of GI symptoms was found to be lower during physical exercise training session than in the rest state, suggesting that physical exercise may act as a modulator of GI function. Furthermore, there were no difference in frequency and intensity of GI symptoms between moderate and vigorous physical exercise groups.

The predisposition to present some type of GI symptoms depends on factors such as the type of sport practiced, duration and intensity of the physical exercise, age, training status, gender, and type of diet. ${ }^{8}$ With regards to lower GI symptoms, diarrhea affects $8 \%$ to $54 \%$ whereas abdominal cramps (lower abdominal pain), blood in stools and flatulence affect $33 \%, 2 \%$ to $16 \%$, and $38 \%$ of athletes, respectively. ${ }^{27,28}$ In the present study, the respondents reported diarrhea (36.6\% and 26.1\%), abdominal cramps (lower abdominal pain) (40.8\% and 21.8\%), presence of blood in stools (6.3\% and 3.5\%), and bloating (64.1\% and $29.6 \%$ ), at rest and during physical exercise training session, respectively. Except for flatulence, the prevalences of GI symptoms found in the present study were similar to those described in the literature for endurance athletes.

Considering upper GI symptoms in athletes, loss of appetite is experienced by $12 \%$ to $50 \%$, eructation $12 \%$ to $36 \%$, nausea $4 \%$ to $21 \%$, vomiting $2 \%$ to $31 \%$, and heartburn by $8 \%$ to $10 \% .{ }^{17,29,30}$ In the present study, $18.3 \%$ and $14.1 \%$ reported loss of appetite; $73.9 \%$ and $52.1 \%$ eructation; $31.0 \%$ and $25.4 \%$ nausea; $7.0 \%$ and $0.7 \%$ vomiting; and $50.7 \%$ and $42.3 \%$ heartburn, at rest and during physical exercise, respectively. Except for loss of appetite and vomiting, the prevalence of GI symptoms found in the present study were similar to those described by the literature for endurance athletes. On the comparison of the prevalence of lower and upper GI symptoms, except for vomiting, upper GI symptoms were more prevalent. These results are in 
Table 4. Comparison of Weekly Intensity Index of GI Symptoms during Rest and Physical Exercise

\begin{tabular}{|c|c|c|c|c|c|c|c|}
\hline \multirow{3}{*}{ Symptom } & \multicolumn{4}{|c|}{ Group } & \multirow{3}{*}{$\begin{array}{c}P \text {-value } \\
\text { (intensity) } \\
\text { (moderatex } \\
\text { vigorous) }\end{array}$} & \multirow{3}{*}{$\begin{array}{c}P \text {-value } \\
\text { (state) } \\
\text { (rest } \times \text { physical } \\
\text { exercise) }\end{array}$} & \multirow{3}{*}{$\begin{array}{c}P \text {-value } \\
\text { (interaction) } \\
\text { (intensity } x \\
\text { state) }\end{array}$} \\
\hline & \multicolumn{2}{|c|}{ Moderate $(n=71)$} & \multicolumn{2}{|c|}{ Vigorous $(n=71)$} & & & \\
\hline & Rest & $\begin{array}{l}\text { Physical } \\
\text { exercise }\end{array}$ & Rest & $\begin{array}{l}\text { Physical } \\
\text { exercise }\end{array}$ & & & \\
\hline Epigastric pain & $1.89 \pm 1.40$ & $1.41 \pm 0.90$ & $1.86 \pm 1.30$ & $1.59 \pm 1.13$ & 0.587 & 0.009 & 0.459 \\
\hline Regurgitation & $1.63 \pm 1.00$ & $1.52 \pm 0.86$ & $1.87 \pm 1.47$ & $1.68 \pm 1.40$ & 0.172 & 0.283 & 0.769 \\
\hline Nausea & $1.54 \pm 0.95$ & $1.52 \pm 0.89$ & $1.66 \pm 1.01$ & $1.39 \pm 0.80$ & 1.000 & 0.197 & 0.246 \\
\hline Vomiting & $1.11 \pm 0.65$ & $1.00 \pm 0.00$ & $1.13 \pm 0.51$ & $1.04 \pm 0.36$ & 0.595 & 0.064 & 0.791 \\
\hline Early satiety & $2.58 \pm 1.65$ & $1.83 \pm 1.42$ & $1.96 \pm 1.21$ & $1.59 \pm 1.01$ & 0.007 & 0.001 & 0.234 \\
\hline Dysphagia & $1.06 \pm 0.37$ & $1.08 \pm 0.41$ & $1.31 \pm 0.98$ & $1.24 \pm 0.92$ & 0.018 & 0.806 & 0.567 \\
\hline Bloating & $2.27 \pm 1.32$ & $1.56 \pm 0.95$ & $2.62 \pm 1.39$ & $1.66 \pm 1.07$ & 0.113 & $<0.001$ & 0.372 \\
\hline Eructation & $2.30 \pm 1.14$ & $1.69 \pm 0.94$ & $2.50 \pm 1.36$ & $2.03 \pm 1.16$ & 0.050 & $<0.001$ & 0.627 \\
\hline Abdominal noise & $2.70 \pm 1.15$ & $1.73 \pm 0.99$ & $2.82 \pm 1.26$ & $2.07 \pm 1.19$ & 0.100 & $<0.001$ & 0.410 \\
\hline Abdominal cramps & $1.82 \pm 1.15$ & $1.44 \pm 0.98$ & $1.96 \pm 1.24$ & $1.46 \pm 0.88$ & 0.506 & 0.001 & 0.658 \\
\hline Flatulence & $2.93 \pm 1.05$ & $2.21 \pm 1.11$ & $3.06 \pm 1.35$ & $2.45 \pm 1.26$ & 0.199 & $<0.001$ & 0.692 \\
\hline Blood in stools & $1.04 \pm 0.20$ & $1.01 \pm 0.12$ & $1.11 \pm 0.46$ & $1.08 \pm 0.44$ & 0.082 & 0.486 & 1.000 \\
\hline Diarrhea & $1.65 \pm 1.14$ & $1.32 \pm 0.81$ & $1.68 \pm 1.05$ & $1.48 \pm 0.89$ & 0.432 & 0.026 & 0.586 \\
\hline Constipation & $1.94 \pm 1.46$ & $1.58 \pm 1.22$ & $2.20 \pm 1.55$ & $1.72 \pm 1.27$ & 0.229 & 0.010 & 0.731 \\
\hline Steatorrhea & $1.07 \pm 0.31$ & $1.04 \pm 0.20$ & $1.04 \pm 0.20$ & $1.00 \pm 0.00$ & 0.160 & 0.160 & 0.778 \\
\hline Mucus in stools & $1.18 \pm 0.46$ & $1.13 \pm 0.38$ & $1.30 \pm 0.78$ & $1.13 \pm 0.48$ & 0.384 & 0.083 & 0.384 \\
\hline Loss of appetite & $1.38 \pm 0.99$ & $1.41 \pm 1.04$ & $1.28 \pm 0.72$ & $1.28 \pm 0.70$ & 0.279 & 0.892 & 0.892 \\
\hline Heartburn & $1.96 \pm 1.16$ & $1.82 \pm 1.02$ & $2.11 \pm 1.42$ & $1.77 \pm 1.12$ & 0.690 & 0.091 & 0.486 \\
\hline
\end{tabular}

Values are presented as mean \pm SD.

line with those of Sullivan, ${ }^{29}$ who also found that upper GI symptoms were more prevalent during the rest state and in sedentary individuals ${ }^{29}$ and that lower GI symptoms were more prevalent in endurance athletes. ${ }^{14,29}$ Therefore, it is reasonable to suppose that the higher prevalence of upper than lower GI symptoms found in the present study might be due to the fact that participants were not elite athletes, thus more closely resembling sedentary individuals than elite athletes from a functional perspective.

Although the prevalence of GI symptoms in our sample proved similar to rates in athletes performing high intensity endurance activities, the symptoms reported by individuals in this study were of very light intensity both at rest and during physical exercise training session. These findings are in accordance with our hypothesis. Furthermore, comparison of weekly frequency and intensity index of GI symptoms at rest versus during physical exercise training session revealed symptoms with higher frequency and intensity during rest, suggesting that physical exercise may act as a regulator of the GI system. Indeed, a plethora of studies showed that physical exercise may influence the GI tract function by decrease GI blood flow, increase in GI motility, increase mechanical bouncing and neuro-immuno-endocrine alterations. ${ }^{31-33}$ Many hormones associated with GI function at rest (secretion, absorption, and motility) alter during exercise, in terms of plasma concentrations: cholecystokinin, vasoactive intestinal peptide, secretin, pancreatic polypeptide, somatostatin, peptide histidine isoleucine, peptide YY, gastrin, glucagon, motilin, catecholamines, endorphins, and prostaglandins. ${ }^{33-35}$

Pugh et al ${ }^{36}$ reported for the first time that acute high intensity interval running $(18 \times 400 \mathrm{~m}$ interval efforts at $120 \%$ of maximum oxygen uptake interspaced by running at a velocity associated with $50 \%$ of maximum oxygen uptake for an amount of time equal to $75 \%$ of that taken to run the $400 \mathrm{~m}$ ) increases intestinal cellular damage and GI permeability in 11 trained male runners though such markers do not correlate to symptoms of GI discomfort; however, the low scores for GI discomfort found by the authors may be explained due to the fact that the individuals were healthy trained males and had no history of GI disease. Moreover, they were also well hydrated and ha- 
bituated to the high intensity interval running.

A few limitations of the present study must be mentioned. First, as for all studies employing questionnaires, the present results rely on the honesty and level of recall of respondents. Second, the present study was that type of diet was not evaluated, a factor that can impact GI function. Third, we assessed volunteers that practiced a great variety of exercise (e.g., running and resistance training). Fourth, the intensity of physical exercise used in the analysis was reported by volunteers it was not possible to determine the true training status of the individuals. Fifth, we did not ask about GI disease, such as lactose intolerance, celiac disease, or IBS. Sixth, we did not assess symptoms that occurred in the first few hours after exercise. Seventh, volunteers were recruited over the internet thus forming a convenience sample. In this context, interest in participating in the study may have been higher among those who had already developed GI symptoms. To minimize this problem, the study was made known on social media and the University website. Therefore, we believe that the large number of respondents diluted this potential bias. Nevertheless, we believe that these limitations do not prevent conclusions being drawn from the study because we use a "ecological validity" approach.

The use of internet-based questionnaire has several methodological advantages: the interface is user-friendly, the questionnaire can be answered in 5 to 10 minutes and by people from different regions, the analysis is quick and easy because all the results can be placed directly into a database, the questionnaire guides the respondent, and therefore prevents incorrect and/or inconsistent answers, and there is no need to interpret erasures.

Most previous studies evaluated 3 to 5 GI symptoms whereas this study 18 GI symptoms were evaluated. We also evaluated the frequency and intensity of GI symptoms of physically active individuals while performing the physical exercise compared to the rest state while most other studies have evaluated GI symptoms only during the practice of physical exercise. Additionally, we evaluated subjects who performed different modalities of physical exercise while previous studies investigated athletes that performed only endurance physical exercise. Thus, we believe that the results of this study represent a breakthrough for exercise physiology applied to the GI tract, showing that not only endurance athletes suffer from GI symptoms.

Finally, this study proposes a method for assessing frequency and intensity of GI symptoms. In the literature, to the best of our knowledge, there is no current definition of what constitutes a severe or non-severe symptom in exercisers individuals. Therefore, the questionnaire used in this study may represent a useful approach for categorizing symptoms.

In summary, our data indicated that physical exercise performed by exercisers individual can trigger GI symptoms. However, the symptoms in the present sample were of very light intensity. Furthermore, several GI symptoms in exercisers individuals were more prevalent during rest than physical exercise for both moderate and vigorous groups, suggesting that physical exercise may act as a regulator of the GI tract.

\section{FINANCIAL SUPPORT}

This study was funded by Fundação de Amparo à Pesquisa do Estado de Goiás-FAPEG/Brazil.

\section{CONFLICT OF INTEREST}

No potential conflict of interest relevant to this article was reported.

\section{AUTHOR CONTRIBUTION}

Conceptualization: de Lira CA, Andrade MS, Vancini RL. Methodology: de Lira CA, Viana RB, Mesquista KP, Santos DA. Formal analysis: Viana RB, Santos DA. Funding acquisition: de Lira CA. Project administration: de Lira CA. Writing - original draft: de Lira CA, Viana RB, Santos DA. Writing - review and editing: de Lira CA, Viana RB, Santos DA, Campos MH, Andrade MS, Vancini RL. Approval of final manuscript: all authors.

\section{ORCID}

de Lira CA https://orcid.org/0000-0001-5749-6877 Viana RB https://orcid.org/0000-0001-9200-3185 Mesquista KP https://orcid.org/0000-0003-2055-8282

Santos DA https://orcid.org/0000-0002-7664-5468 Campos MH https://orcid.org/0000-0002-4724-2221

Andrade MS https://orcid.org/0000-0002-7004-4565

Vancini RL https://orcid.org/0000-0003-1981-1092

\section{ACKNOWLEDGEMENTS}

We would like to thank the participants for their effort and commitment to the research project. 


\section{REFERENCES}

1. Garber CE, Blissmer B, Deschenes MR, et al. American College of Sports Medicine position stand. Quantity and quality of exercise for developing and maintaining cardiorespiratory, musculoskeletal, and neuromotor fitness in apparently healthy adults: guidance for prescribing exercise. Med Sci Sports Exerc 2011;43:1334-1359.

2. Pedersen BK, Saltin B. Exercise as medicine: evidence for prescribing exercise as therapy in 26 different chronic diseases. Scand J Med Sci Sports 2015;25 Suppl 3:1-72.

3. Costa RJ, Snipe RM, Kitic CM, Gibson PR. Systematic review: exercise-induced gastrointestinal syndrome-implications for health and intestinal disease. Aliment Pharmacol Ther 2017; 46:246-265.

4. de Lira CA, Vancini RL, Ihara SS, da Silva AC, Aboulafia J, Nouailhetas VL. Aerobic exercise affects C57BL/6 murine intestinal contractile function. Eur J Appl Physiol 2008;103:215-223.

5. Lira CAB de, Vancini RL, Silva AC da, Nouailhetas VLA. Efeitos do exercício físico sobre o trato gastrintestinal. Rev Bras Med Esporte 2008;14:64-67.

6. Strid H, Simrén M, Störsrud S, Stotzer PO, Sadik R. Effect of heavy exercise on gastrointestinal transit in endurance athletes. Scand J Gastroenterol 2011;46:673-677.

7. Waterman JJ, Kapur R. Upper gastrointestinal issues in athletes. Curr Sports Med Rep 2012;11:99-104.

8. Peters HP, De Vries WR, Vanberge-Henegouwen GP, Akkermans LM. Potential benefits and hazards of physical activity and exercise on the gastrointestinal tract. Gut 2001;48:435439.

9. de Oliveira EP, Burini RC. The impact of physical exercise on the gastrointestinal tract. Curr Opin Clin Nutr Metab Care 2009;12:533-538.

10. de Oliveira EP, Burini RC, Jeukendrup A. Gastrointestinal complaints during exercise: prevalence, etiology, and nutritional recommendations. Sports Med 2014;44 Suppl 1:S79-S85.

11. Peters HP, Bos M, Seebregts L, et al. Gastrointestinal symptoms in long-distance runners, cyclists, and triathletes: prevalence, medication, and etiology. Am J Gastroenterol 1999;94: 1570-1581.

12. Peters HP, Zweers M, Backx FJ, et al. Gastrointestinal symptoms during long-distance walking. Med Sci Sports Exerc 1999; 31:767-773.

13. Simrén M. Physical activity and the gastrointestinal tract. Eur J Gastroenterol Hepatol 2002;14:1053-1056.

14. Sullivan SN, Wong C, Heidenheim P. Does running cause gas- trointestinal symptoms? A survey of 93 randomly selected runners compared with controls. NZ Med J 1994;107:328-331.

15. ter Steege RW, Van der Palen J, Kolkman JJ. Prevalence of gastrointestinal complaints in runners competing in a long-distance run: an internet-based observational study in 1281 subjects. Scand J Gastroenterol 2008;43:1477-1482.

16. van Nieuwenhoven MA, Brouns F, Brummer RJ. Gastrointestinal profile of symptomatic athletes at rest and during physical exercise. Eur J Appl Physiol 2004;91:429-434.

17. Riddoch C, Trinick T. Gastrointestinal disturbances in marathon runners. Br J Sports Med 1988;22:71-74.

18. Wilson PB. Dietary and non-dietary correlates of gastrointestinal distress during the cycle and run of a triathlon. Eur J Sport Sci 2016;16:448-454.

19. Costa RJ, Miall A, Khoo A, et al. Gut-training: the impact of two weeks repetitive gut-challenge during exercise on gastrointestinal status, glucose availability, fuel kinetics, and running performance. Appl Physiol Nutr Metab 2017;42:547-557.

20. Collings KL, Pierce Pratt F, Rodriguez-Stanley S, Bemben M, Miner PB. Esophageal reflux in conditioned runners, cyclists, and weightlifters. Med Sci Sports Exerc 2003;35:730-735.

21. Pugh JN, Kirk B, Fearn R, Morton JP, Close GL. Prevalence, severity and potential nutritional causes of gastrointestinal symptoms during a marathon in recreational runners. Nutrients 2018;10:E811.

22. Ho SS, Radavelli-Bagatini S, Dhaliwal SS, Hills AP, Pal S. Resistance, aerobic, and combination training on vascular function in overweight and obese adults. J Clin Hypertens (Greenwich) 2012;14:848-854.

23. Many G, Hurtado ME, Tanner C, et al. Moderate-intensity aerobic training program improves insulin sensitivity and inflammatory markers in a pilot study of morbidly obese minority teens. Pediatr Exerc Sci 2013;25:12-26.

24. Naves JP, Viana RB, Rebelo AC, et al. Effects of high-intensity interval training vs. sprint interval training on anthropometric measures and cardiorespiratory fitness in healthy young women. Front Physiol 2018;9:1738.

25. Maron BJ, Zipes DP, Kovacs RJ. Eligibility and disqualification recommendations for competitive athletes with cardiovascular abnormalities: preamble, principles, and general considerations: a scientific statement from the American Heart Association and American College of Cardiology. J Am Coll Cardiol 2015;66:2343-2349.

26. Likert R. A technique for the measurement of attitudes. Arch Psychol 1932;22:55.

27. Gil SM, Yazaki E, Evans DF. Aetiology of running-related gas- 
trointestinal dysfunction. How far is the finishing line? Sports Med 1998;26:365-378.

28. Jeukendrup AE, Vet-Joop K, Sturk A, et al. Relationship between gastro-intestinal complaints and endotoxaemia, cytokine release and the acute-phase reaction during and after a long-distance triathlon in highly trained men. Clin Sci (Lond) 2000;98:47-55.

29. Sullivan SN. Exercise-associated symptoms in triathletes. Phys Sportsmed 1987;15:105-108.

30. Worobetz LJ, Gerrard DF. Gastrointestinal symptoms during exercise in Enduro athletes: prevalence and speculations on the aetiology. NZ Med J 1985;98:644-646.

31. Moses FM. The effect of exercise on the gastrointestinal tract. Sports Med 1990;9:159-172.

32. Peters HP, Akkermans LM, Bol E, Mosterd WL. Gastrointesti- nal symptoms during exercise: the effect of fluid supplementation. Sports Med 1995;20:65-76.

33. Brouns F, Beckers E. Is the gut an athletic organ? Digestion, absorption and exercise. Sports Med 1993;15:242-257.

34. O'Connor AM, Johnston CF, Buchanan KD, Boreham C, Trinick TR, Riddoch CJ. Circulating gastrointestinal hormone changes in marathon running. Int J Sports Med 1995;16:283-287.

35. Demers LM, Harrison TS, Halbert DR, Santen RJ. Effect of prolonged exercise on plasma prostaglandin levels. Prostaglandins Med 1981;6:413-418.

36. Pugh JN, Impey SG, Doran DA, Fleming SC, Morton JP, Close GL. Acute high-intensity interval running increases markers of gastrointestinal damage and permeability but not gastrointestinal symptoms. Appl Physiol Nutr Metab 2017;42:941-947. 